\title{
The Diagnostic Difficulties and The Delayed Identification of Anaplastic Large Cell Lymphoma in A Teenage Patient. A Review of Literature
}

\author{
Bartosz Pogorzały $^{1}$, Paweł Kurzawa ${ }^{2,3 \#}$, Grzegorz Mielniczek ${ }^{1}$, Michał Dopierała ${ }^{4}$, Swati Singh ${ }^{5}$ \\ and Katarzyna Derwich ${ }^{1 *}$
}

${ }^{1}$ Department of Pediatric Oncology, Hematology and Transplantology, Poznan University of Medical Sciences, Poland ${ }^{2}$ Department of Clinical Pathology, Poznań University of Medical Sciences, Poland

${ }^{3}$ Department of Oncological Pathology, University Hospital of Lord's Transfiguration, Partner of Karol Marcinkowski University of Medical Sciences, Poland

${ }^{4}$ Department of Pathology, Children's Hospital, Poznan, Poland

${ }^{5}$ Center for Medical Education in English, Poznan University of Medical Sciences, Poland

*Corresponding author: Katarzyna Derwich, Department of Pediatric Oncology, Hematology and Transplantology, Poznan

University of Medical Sciences, Poznan 60-572, Szpitalna 27/33, Poland

\#co-first author: Paweł Kurzawa, Department of Clinical Pathology, Poznań University of Medical Sciences, Poland

\section{ARTICLE INFO}

Received: 蔍 July 27, 2021

Published: 幽 August 05, 2021

Citation: Bartosz Pogorzały, Grzegorz Mielniczek, Paweł Kurzawa, Michał Dopierała, Swati Singh, Katarzyna Derwich. The Diagnostic Difficulties and The Delayed Identification of Anaplastic Large Cell Lymphoma in A Teenage Patient. A Review of Literature. Biomed J Sci \& Tech Res 37(5)-2021. BJSTR. MS.ID.006067.

\section{ABSTRACT}

Systemic anaplastic large cell lymphoma (S-ALCL) is a rare and aggressive lymphoma. In most cases it arises in cervical and axillary lymph nodes. At times, the disease may have an unusual course when the primary site appears in other locations, like skin, bone, lung liver. This may lead to diagnostic dilemmas. We report an unusual case of a 16-year-old boy with S-ALCL who presented with a nodular skin lesion located in the lower abdomen. Initially, based on the physical examination, he was misdiagnosed with an abscess. This resulted in the omission of diagnosing the neoplasm and caused the development of a systemic disease The diagnosis of ALCL is established in an advanced stage. This process relies on a histopathological and complex immunohistochemical examination of the collected material. To provide the best adjusted therapy, there is a requirement of a precise identification of neoplastic cells' immunophenotype. It should be performed in the facility with the highest reference level. In the view of above, patient's life depends on fast and exact diagnostic. Specialists, who are usually the first to encounter such patients, are crucial to initiate the relevant diagnostic processes and subsequently send the patient to the oncology unit.

Keywords: Anaplastic Large Cell Lymphoma; Non-Hodgkin Lymphoma; Lymph Node; Skin Lesion; Disseminated Neoplasm; Biopsy; Misdiagnosis

\section{Introduction}

Anaplastic large cell lymphoma (ALCL) originates from peripheral T-lymphocytes and is the most common mature T-cell neoplasm in children and adolescents. All in all, these neoplasms are relatively rare among all types of lymphomas. ALCL comprises about $10-20 \%$ of all non-Hodgkin lymphomas (NHL) in the above group of patients [1-3]. Essentially, this lymphoma does not occur frequently and is often difficult to distinguish, though its identification is essential for both the prognosis and treatment [4]. The primary site of the lymphoma can appear in almost any location, including lymph nodes. This may result in unspecific clinical signs of disease leading to why it can take several months from the appearance of the first symptoms to make an accurate diagnosis. 
ALCL was recently classified by the World Health Organization (WHO) as primary cutaneous ALCL (C-ALCL) and systemic ALCL (S-ALCL). The second category was further divided into two distinct types based on expression of a protein called anaplastic lymphoma kinase (ALK), ALCL ALK-positive (ALK+) and ALCL ALKnegative (ALK-) [5]. The presence of this protein on neoplastic cells is more frequent in children and young adults, with a median age of approximately 30 years, whereas the lack of it is proven in older adults (median age of about 55 years). Simultaneously, this feature is associated with improved overall survival $[2,6,7]$. Additionally, the assessment of ALK expression plays an essential role in the case of S-ALCL. The overall survival rate of ALK+ lymphoma increases up to $70 \%$ and is far better that of ALK- lymphoma that ranges from $15-45 \%[6,7]$. C-ALCL is marked by indolent development and a favorable prognosis with a survival rate of $80-95 \%$ [2]. We present the case of a patient with an unusual clinical manifestation and difficulties in the diagnostics of S-ALCL. We would like to point out what to do in order to avoid mistakes in the early stages of this disease. Additionally, attention should be paid to the aggressive and insidious growth of S-ALCL in a very brief time.

\section{Case Presentation}

\section{Patient History and Information}

A sixteen-year-old boy was admitted to the Surgery Clinic in July 2017 due to a nodular skin lesion. The $4 \mathrm{~cm}$ lesion was located in his lower abdomen and was darker than the surrounding skin. On clinical examination, the lesion was suspected to be an abscess and the patient was sent to surgery. The lesion was incised and did not contain any liquid content. No tissue material was taken for histopathological examination. The wound did not heal, was suppurating and slowly reducing its size. Furthermore, in August 2017, hard and painless tumors appeared in the area of both groins. Ultrasound (US) and computed tomography (CT) of the abdominal cavity and pelvis showed enlarged lymph nodes in the inguinal and pelvic regions. Based on the above examination, lymphoma was suspected, and the boy was sent to the Department of Pediatric Oncology, Hematology and Transplantology at Poznan University of Medical Sciences.

\section{Diagnostic Assessment}

On the day of admission in September 2017, the physical examination showed an unhealed wound on the skin of the lower abdomen and palpable hard nodal masses in the iliac fossae. We have only a photographic evidence of the scar 4 months after surgical treatment (Figure 1). Additionally, a painless fistula with leakage was observed inside the oral cavity in the area of the right molar teeth. The liver and spleen were not enlarged. The results of biochemical tests, morphology, and peripheral blood smears were within normal limits. During his stay in the Department, the patient presented with a low-grade fever and increasing facial asymmetry caused by swelling of the right cheek. CT scan and magnetic resonance imaging (MRI) of the head revealed the solid mass (60x42x35 mm) filled almost all of the space of the right maxillary sinus and destroyed the structure of the posterior wall of the sinus, invading the pterygopalatine fossa and adipose tissue, reaching the area of the masseter muscles. Laterally, it was spreading into the right buccal area where from the medial side, it was infiltrating the lateral part of the palate. Upwards, it was penetrating the inferior orbital fissure and damaging the upper wall of the maxillary sinus into the right orbit. There were no signs of the tumor spreading to the middle cranial fossa. Furthermore, numerous enlarged lymph nodes of the upper neck region were found. Biopsies of the inguinal node, exophytic craniofacial tumor, and bone marrow along were performed. The patient was also examined with positron emission tomography (PET), which allowed for a determination of disease in stage IV.

Figure 1: The scar in the lower abdomen 4 months after surgical treatment of the primary lesion. 


\section{Histopatological Findings}

Materials and Methods: The specimen was taken from three different sites and sent to Pathology Department. The first surgery consisted of excisional biopsy of enlarged, inguinal lymph node. 5 days later the biopsy of bone marrow (two trephines) was done. 6 days later excisional biopsy of two tumors in the oral cavity, (including right maxilla and oral vestibule) was performed. All resected specimens were fixed in $10 \%$ buffered formalin, routinely processed and embedded in paraffin, stained with hematoxylin and eosin. An initial panel of immunohistochemical stains was performed on an automated immunostainer (Ventana Benchmark, Roche/Ventana Medical Systems, Tucson, AZ) and this included: CD30, EMA (Epithelial Membrane Antigen), ALK-1, CD43, CD3, CD15, CD20, Desmina (DES), CD25, Granzyme B, Perforin, TIA 1, CD7, CD5, Cd8, Cd56, MUM 1, PDL 1, CD4, CD2, EBI-1, PAX-5, Ki 67.

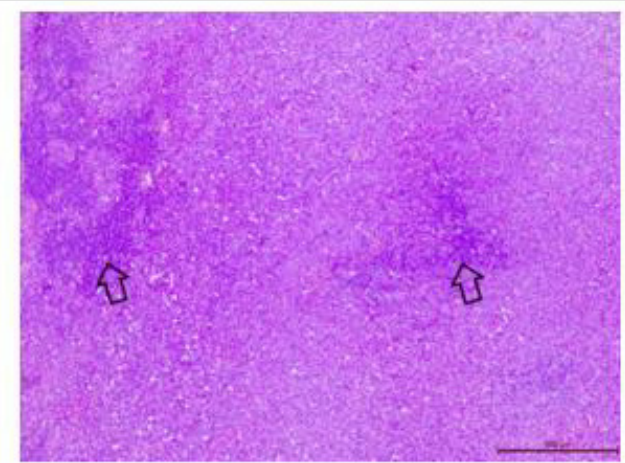

(a)

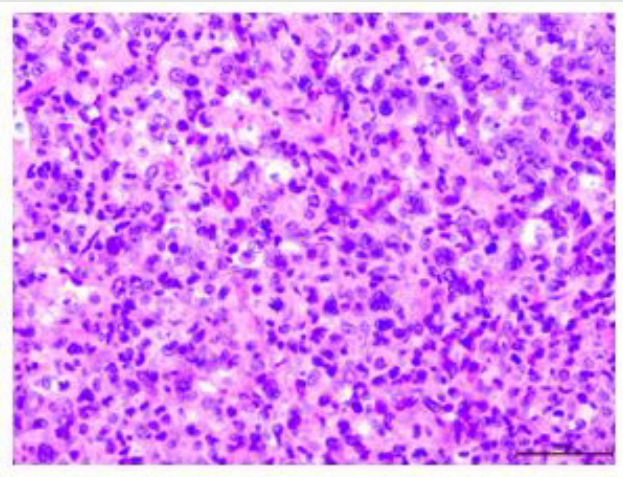

(b)

Figure 2: (a) The tumor invades almost entire inguinal lymph node tissue. There are only small areas of the normal lymph node (arrows), H\&E, 200X; (b) The neoplastic cells range from small to large ones. They usually have large, irregular, and bizarre or polylobated nuclei, H\&E ,400X.

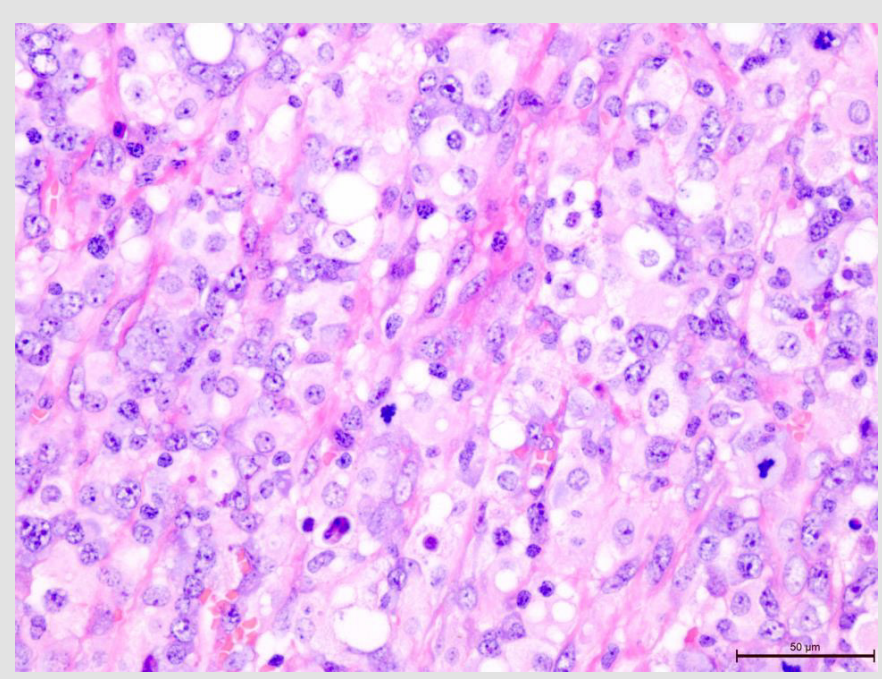

Figure 3: The neoplastic cells infiltrating the mucosa of the oral cavity, H\&E, 400X.

Results: Grossly, the first resected specimen consisted of solid soft tissue in form of a lymph node measuring 3,5 cm x 3,0 cm x $2,0 \mathrm{~cm}$. It was oval in shape, but the external surface was rather smooth. On cross section, the tumor was white to grey, solid and encapsulated. The second specimen consisted of two core biopsies specimen of bone marrow measuring 2,6 cm and 0,8 cm in length. The third specimen from the oral cavity consisted of two separated specimens each of which was fragmented measuring 5 $\mathrm{cm}$ and $3 \mathrm{~cm}$ respectively in greatest diameter. Microscopically, the neoplasm invaded the lymph node and the specimens from oral cavity. The bone marrow specimen was not impaired. The tumor cell occupied almost entire parenchyma of the lymph node (Figure 
2a). The neoplastic cells differed in size from small to large ones (Figure 2b). This was helpful in distinguishing ALCL from Hodgkin Lymphoma. The cells invading the lymph node and the oral cavity had the same morphology (Figure 3). Most of the neoplastic cells were large, irregular and bizarre. They often had polylobated nuclei and the subset of cells had eccentric horse- or kidney-shaped nuclei consisted with Hallmark cells characteristic of ALCL. Admixed with the tumor were scattered small lymphocytic cells. Mitotic figures were easily encountered (60 per 10 high power fields). There was no necrosis.
Immunohistochemically the tumor cells stained for CD 30 and ALK 1 showing nuclear and cytoplasmic staining (Figure 4a and $4 \mathrm{~b})$. Such pattern of staining correlates with underlying genetic abnormality $t(2 ; 5)$. They also stained for EMA, CD43, CD25, Granzyme B, Perforin, TIA 1, Ki -67 in $80 \%$ of the cells and focally for CD3, CD7, CD5, CD8, CD56, MUM 1, PDL 1. They neoplastic cells were negative for CD15, CD20, Desmin, CD4, CD2, EBI-3, PAX 5. The microscopic and immunophenotypic studies confirmed the diagnosis of Anaplastic Large Cell Lymphoma, common type.

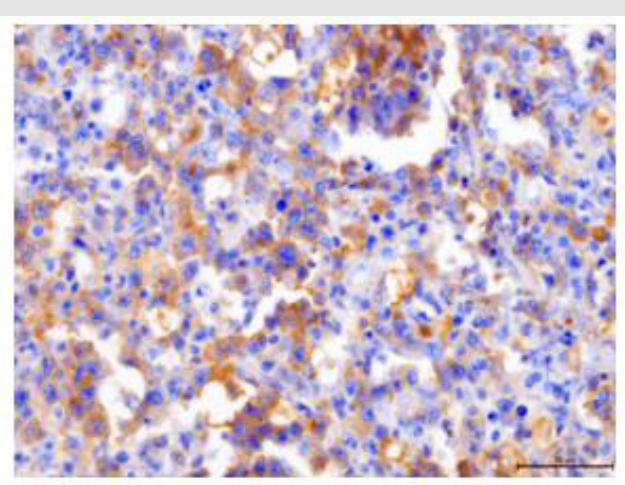

(a)

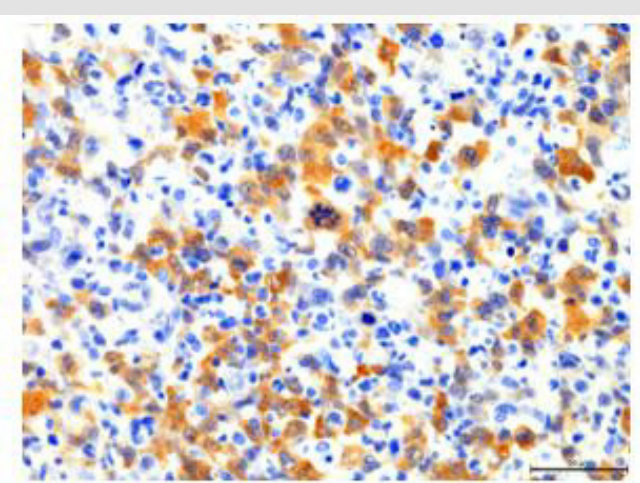

(b)

Figure 4: (a) The tumor cells are diffusely positive for CD30, 400X; (b) Most of the neoplastic cells stain for ALK 1, 400X.

\section{Diagnosis}

Based on the PET scan and pathology report, ALCL in stage IV was diagnosed.

\section{Treatment and Outcome}

After establishing the final diagnosis, treatment according to the international protocol ALCL 99 was started. It consisted of six alternating, consecutive courses of AM (dexamethasone, methotrexate, ifosfamide, cytarabine, etoposide) and BM (dexamethasone, methotrexate, cyclophosphamide, doxorubicin) [8]. Currently, the patient remains in the first complete remission of the disease, 36 months after their treatment.

\section{Discussion}

S-ALCL remains a moderately aggressive lymphoproliferative neoplasm with worse outcomes than most B-cell lymphomas, yet significantly better survival than other peripheral T-cell lymphomas [9]. The majority of patients are male and present with advanced disease in stages III or IV, often with systemic symptoms, especially fever. Dissimilar from adults, ALCL in children usually demonstrates expression of ALK $[1,3,6,7]$. The diagnosis of S-ALCL can be challenging, mainly when first signs appear in the skin. One of the reasons for this is that both types of S-ALCL can secondarily involve the skin and C-ALCL can secondarily involve regional lymph nodes. It shows the importance of the correlation of histopathological findings with staging and other clinical data in order to establish a proper diagnosis [9]. The stage of the disease at the beginning is frequently determined as advanced. It may often result from both a late diagnosis as well as a rather rapid developmental rate of disseminated neoplastic cells. In literature, such cases take a few months from the first signs of disease (Table 1) [10-15]. In our case, that period lasted three months.

The primary site of S-ALCL can occur in almost any location in the human body. In most cases at first, it affects lymph nodes, and the initial sign is painless lymphadenopathy. If that happens, the proper procedure is to carry out a broad differential diagnosis. We should take into account bacterial and viral infections, zoonotic diseases, connective tissue diseases and neoplasms, notably leukemia and lymphomas. In $60 \%$ of ALK+ anaplastic large cell lymphomas, there is an additional involvement of extranodal tissues most often concerning skin, bones, bone marrow, soft tissues, lungs and liver $[1,6,7]$. However, S-ALCL presenting initially as a localized skin lesion is an infrequent finding, as was the case with our patient $[11,15]$. His first sign of lymphoma was a nodular 
skin lesion that resembled an abscess. Other primary extranodal localizations of S-ALCL are presented in literature, which include soft tissues, lungs, bones, stomach, small intestine, tonsils, and the central nervous system [13,16-18].

Symptoms resulting from extranodal involvement (listed in Table 1) may imitate various other diseases and linking them to a lymphoproliferative neoplasm can be quite challenging. Examples of an atypical clinical course of ALCL can be found in literature $[10,11,13,14,19]$. In cases of involved lungs, the lesion may present as tuberous masses, atelectasis, displacement of the mediastinum to one side and pleural effusion [14]. Sites in the central nervous system are accompanied by various neurological disorders such as diplopia, nausea, vomiting, neck stiffness, persistent headaches, impairment of recent memory, disorientation regarding the time and place and poor state of mind $[10,13]$. Skin lesions can occur either as a soft and swollen nodular lesion or as a scattered, erythematous and maculopapular one, both of which can display ulceration [10, 11]. Moreover, a case of incorrect diagnosis of mononucleosis has been reported in a teenage girl with a nasopharyngeal mass and cervical lymphadenopathy where the mass caused nasal airway obstruction [19].

Table 1: The review of the approximate time from the first presenting signs to diagnosis and distinctive symptoms resulting from particular extranodal sites of ALCL in the literature.

\begin{tabular}{|c|c|c|}
\hline Localization & Time from the First Signs to Diagnosis & Specific Extranodal Symptoms \\
\hline Skin lesions - other cases $[10,11,15]$ & $2-3$ months & $\begin{array}{c}\text { diffuse and irregular areas with scalp swelling, scattered } \\
\text { erythematous macropapules, reddish-purple nodules, erosive } \\
\text { nodule, }\end{array}$ \\
\hline Central nervous system [10,13,17] & $3-4$ months & $\begin{array}{c}\text { headache, seizures, diplopia, vomit, runny nose, impaired recent } \\
\text { memory, disorientation regarding the time and place }\end{array}$ \\
\hline Nasopharynx [19] & 3 months & anosmia, persistent nasal airway obstruction, muffled voice \\
\hline Lungs [14] & 4 months & $\begin{array}{c}\text { abdominal and periumbilical pain, constipation, abdominal } \\
\text { distension, sore throat, odynophagia, dysphagia, dysphonia }\end{array}$ \\
\hline Gastrointestinal tract [18] & from a week to a few months & microscopic hematuria, pyuria, increased, urinary frequency \\
\hline Urinary bladder [12] & 39 days &
\end{tabular}

Due to a wide range of complaints, patients may visit many specialists in the early stages of disease. These physicians, who are usually not oncologists, should keep oncological alertness in every situation including trivial ones, as they play a particularly important role in the initiation of the diagnostic process and referral of patients to oncologists. The proper proceedings have a decisive influence on prognosis, which also includes surgical interventions of precarious lesions. For the described patient, a surgeon made a significant mistake at the beginning of the diagnostic process. Without any examination and histopathological analysis, the doctor considered a nodular skin lesion as an abscess and incised those changes to drain it. Thus, the time to make a diagnosis of ALCL and initiating the treatment in the presented patient was markedly protracted. The absence of any liquid content inside the skin lesion should have led the physician to verify the previous assessment. Moreover, he ought to have collected some material for histopathological examination. What is more, the increased healing time of this wound should have awaken the doctors' suspicion of the neoplastic character of this lesion.

Its histopathological features cause further difficulties in diagnosing ALCL. The marked cellular pleomorphism and morphologic variation of ALCL raises a broad differential diagnosis including cancer, melanoma, sarcoma and other hematopoietic neoplasms such as diffuse large B-cell lymphoma, classical Hodgkin lymphoma, myeloid sarcoma and peripheral T-cell lymphoma not otherwise specified [20]. ALCL shows a broad morphological spectrum. Still, all cases contain a variable percentage of cells with eccentric, horseshoe- or kidney-shaped nuclei, often with an eosinophilic region near the nucleus, socalled hallmark cells [3]. It is essential to recognize that rare cases may have subtle morphological features, including a prominent reactive background [20]. ALK+ and ALK- types of S-ALCL share similar morphological features and diagnosis cannot be established based on morphological evaluation alone. Thus, the immunohistochemistry and molecular profile (IHC) play essential roles in the diagnosis and subclassification of ALCL [9]. The ALK+ form is associated with the NPM-ALK $t(2 ; 5)$ translocation, which highly correlates with the identification of the ALK protein by IHC [2]. CD30 antigen expression in the neoplastic cells is distinctive of ALCL, but it is not a specific marker for this lymphoma. However, the strong and homogenous staining helps distinguish ALCL from other CD30+ lymphomas [6,9]. Besides being CD30+, most cases also express epithelial membrane antigen (EMA). Moreover, ALCLs are considered peripheral T-cell lymphomas as they express one or more T-cell antigens. Therefore, markers like CD2, CD4 and CD5 are positive in a significant proportion of cases. However, due to the loss of several pan T-cell antigens, some cases may have an apparent "null cell" phenotype [6,21]. S-ALCL is not one disease entity, and the "one size fits all" treatment strategy is not ideal [8]. 
To implement the best targeted therapy, there is a necessity of an exact determination of the neoplastic cells' immunophenotype. In view of the above, and due to additionally significant complexity of histopathological diagnostics of lymphomas, this process should be performed in the department of pathology with the highest reference level. The facility should have all available diagnostic techniques, beyond standard pathomorphological evaluation, including but not limited to, broad antibody panels to IHC testing, flow cytometry, cytogenetics and molecular diagnostics [22].

S-ALCL, especially in ALK+ peculiarities, exhibits chemosensitivity in the front line and at relapse, leading to high response rates with vastly different chemotherapy regimens $[9,21]$. The beginning of the treatment based on an incorrect diagnosis may cause the implementation of an incorrect chemotherapy protocol. On the other hand, leaving the patient without any treatment when the disease progresses very quickly can eliminate further therapeutic options and lead to disastrous complications [22]. Results of the Savage et al. study showed that an advanced stage and poor performance status are poor prognostic factors in both ALK+ and ALK- ALCL. In contrast, multiple extranodal sites of involvement and increased age are bad prognostic factors only in the ALK+ type [6]. Implementation of targeted therapy at early stages of disease may result in a better chance of recovery.

\section{Conclusion}

Anaplastic large cell lymphomas represent complicated diagnostic difficulties. The possibility of localization of both primary and secondary sites in extranodal locations makes clinical manifestation non-specific. Presented symptoms may seem trivial or can indicate another condition and as a result, can be easily marginalized. For this reason, it can lead to making a significant mistake at the beginning of the diagnostic process. Complicated pathomorphological diagnostics are based on immunophenotype evaluation with confirmation in the reference facility. As a consequence, ALCL is most often diagnosed in an advanced stage. Physicians who encounter such patients at the beginning are usually not oncologists. However, they are crucial to initiate the relevant diagnostic processes and refer the patient to the oncology center afterward. It is therefore essential to stay alert all of the time. Whether the patient will survive, improve and recover is their responsibility.

\section{Author Contributions}

B.P.: conceptualization, writing - original draft preparation; G.M.: writing - review and editing; P.K. and M.D.: methodology,pictures, editing, resources; J.M.: resources; S.S.: writing - review and editing; K.D.: writing - review and editing, supervision, project administration. All authors have read and agreed to the published version of the manuscript.

\section{Funding}

This research received no external funding.

\section{Informed Consent Statement}

Informed consent was obtained from the patient involved in the study and his legal guardian.

\section{Conflicts of Interest}

The authors declare no conflict of interest.

\section{References}

1. Lowe EJ, Gross TG (2013) Anaplastic large cell lymphoma in children and adolescents. Pediatric Hematology and Oncology 30(6): 509-519.

2. Hapgood G, Savage KJ (2015) The biology and management of systemic anaplastic large cell lymphoma. Blood 126: 17-25.

3. Ferreri AJ, Govi S (2012) Anaplastic large cell lymphoma, ALK-positive. Critical Reviews In Oncology/Hematology 83(2): 293-302.

4. Melissa Pulitzer, Olakunle Ogunrinade, Oscar Lin, Peter Steinherz (2015) ALK-positive (2p23 rearranged) anaplastic large cell lymphoma with localization to the skin in a pediatric patient. Journal of Cutaneous Pathology 42(3): 182-187.

5. Swerdlow SH, Campo E, Harris NL, Jaffe ES, Pileri SA, et al. (2017) WHO Classification of Tumours of Haematopoietic and Lymphoid Tissues. Revised 4th ed. IARC: Lyon, France.

6. Kerry J Savage, Nancy Lee Harris, Julie M Vose, Fred Ullrich, Elaine S Jaffe, Joseph M Connors, et al. (2008) ALK- anaplastic large-cell lymphoma is clinically and immunophenotypically different from both ALK+ ALCL and peripheral T-cell lymphoma, not otherwise specified: report from the International Peripheral T-Cell Lymphoma Project. Blood 111(12): 5496-5504.

7. B Falini, S Pileri, P L Zinzani, A Carbone, V Zagone, et al. (1999) ALK+ lymphoma: clinico-pathological findings and outcome. Blood 93(8): 2697-2706.

8. (2001) An Intergroup Cooperation of the EICNHL. ALCL 99. International Protocol for the Treatment of Childhood Anaplastic Large Cell Lymphoma.

9. Bennani-Baiti N, Ansell S, Feldman AL (2015) Adult systemic anaplastic large cell lymphoma: recommendations for diagnosis and management. Expert Review of Hematology 9(2): 137-150.

10. Hyun-Nam Seo, Jin-Ho Seo, Cheol-Young Lee, Jihye Song, Jong-Hyun Kim, et al. (2017) Cutaneous anaplastic large T-cell lymphoma with invasion of the central nervous system: a case report. Brain tumor research and treatment 5(1): 45-48.

11. Jeong Eun Kim, Eui Hyun Oh, Young Suck Ro, Joo Yeon Ko (2016) CD30positive anaplastic lymphoma kinase-negative systemic anaplastic large-cell lymphoma in a 9-year-old boy. Annals of Dermatology 28(3): 371-374.

12. Haiyan Chen, Yanxia Li, Sucha Nand, Marcus L Quek, Ameet R Kini, Güliz A Barkan (2015) Anaplastic large cell lymphoma involving the urinary bladder: a case report and review of the literature. Diagnostic Cytopathology 43(1): 60-65.

13. Xiaoqin Dong, Jun Li, Na Huo, Yan Wang, Zhao Wu, et al. (2016) Primary central nervous system ALK-positive anaplastic large cell lymphoma in an adult. Medicine (Baltimore) 95(49): e5534.

14. Lu Yu, Lin Li Yan, Shou Jing Yang (2014) Sarcomatoid variant of ALKanaplastic large cell lymphoma involving multiple lymph nodes and 
both lungs with production of proinflammatory cytokines: report of a case and review of literature. International Journal of Clinical and Experimental Pathology 7(8): 4806-4816.

15. AlSayyah A, AlHumidi A (2015) A primary systemic ALCL present initially as cutaneous localized skin lesions: report of an unusual case. The American Journal of Dermatopathology 37(1): e12-14.

16. R D Gascoyne, P Aoun, D Wu, M Chhanabhai, B F Skinnider, T C Greiner, et al. (1999) Prognostic significance of anaplastic lymphoma kinase (ALK) protein expression in adults with anaplastic large cell lymphoma. Blood 93: 3913-3921.

17. Lakshmaiah C Kuntegowdenahalli, Linu Abraham Jacob, Ashok S Komaranchath, Usha Amirtham (2015) A rare case of primary anaplastic large cell lymphoma of the central nervous system. Journal of Cancer Research and Therapeutics 11(4): 943-945.

18. Yi-Ying Lee, Katsuyoshi Takata, Ren-Ching Wang, Sheau-Fang Yang, Shih-Sung Chuang (2017) Primary gastrointestinal anaplastic large cell lymphoma. Pathology 49(5): 479-485.

ISSN: 2574-1241

DOI: 10.26717/BJSTR.2021.37.006067

Katarzyna Derwich. Biomed J Sci \& Tech Res

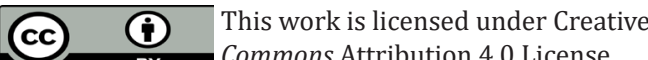

Submission Link: https://biomedres.us/submit-manuscript.php
19. Dion GR, Packer MD (2015) Anaplastic large-cell lymphoma presenting as a nasopharyngeal mass and cervical lymphadenopathy. Ear, Nose \& Throat Journal 94(6): e26-29.

20. Pletneva MA, Smith LB (2014) Anaplastic large cell lymphoma: features presenting diagnostic challenges. Archives of Pathology \& Laboratory Medicine 138(10): 1290-1294.

21. Suzanne D Turner, Laurence Lamant, Lukas Kenner, Laurence Brugières (2016) Anaplastic large cell lymphoma in paediatric and young adult patients. British Journal of Haematology 173(4): 560-572.

22. Walewski (2011) J Rozpoznanie i plan postępowania - podejście do chorego na chłoniaka [Diagnosis and management plan - approach to the lymphoma patient]. In Nowotwory układu chłonnego [Lymphatic system cancers]; Walewski J, (Eds.),; Centrum Medyczne Kształcenia Podyplomowego: Warsaw, Poland, pp. 5-10.

\begin{tabular}{ll} 
BIOMEDICAL & Assets of Publishing with us \\
RESEARCHES & - Global archiving of articles \\
- Immediate, unrestricted online access & - Rigorous Peer Review Process \\
\hline
\end{tabular}

